\title{
Muerte fetal anteparto: ¿es una condición prevenible?
}

\author{
Saulo Molina ${ }^{1}$, Diana Alejandra Alfonso ${ }^{2}$
}

\section{Resumen}

El síndrome de muerte fetal se define como la muerte del feto que se presenta después de la semana 20 de gestación, en ausencia de trabajo de parto instaurado. Constituye un tercio de toda la mortalidad fetal e infantil y más de $50 \%$ de todas las muertes perinatales en los países desarrollados. Las causas de muerte fetal raramente se reportan y la mayoría se desconoce. Se pretende con este artículo hacer una revisión que permita entender la fisiopatología de la entidad, evaluar los factores de riesgo y tratar de brindar a nuestras pacientes la posibilidad de un embarazo exitoso.

Se realizó una revisión de la literatura existente de acuerdo con las bases de datos PubMed, EBSCO, Ovid y Proquest, desde el año 2000 hasta 2008.

A pesar de la disminución significativa de los óbitos fetales en las últimas décadas, sigue siendo un serio problema de salud pública, no sólo por ser una condición prevalente, sino por el subregistro y la ausencia de reportes confiables.

\section{Palabras clave}

Muerte fetal anteparto, óbito fetal, mortalidad perinatal.

\section{Title}

Fetal death syndrome: could it be prevented?

1 Ginecobstetra, Universidad del Rosario, especialista en Medicina Fetal; Medicina Perinatal, Ltda., fellowship en Medicina Materno-Fetal, Hospital de San José, Fundación Universitaria de Ciencias de la Salud; fellowship en Intervención y Terapia Fetal, Baylor College of Medicine-Texas Children's Fetal Center; Coordinador, Unidad de Medicina Materno-Fetal y Fetoscopia, Clínica Colsubsidio; docente, Medicina Materno-Fetal y Ultrasonido, Hospital de San José, Fundación Universitaria de Ciencias de la Salud, Universidad del Rosario y de la Sabana; vicepresidente, Asociación Bogotana de Perinatología, Bogotá, D.C., Colombia.

2 Ginecobstetra, Universidad del Rosario, fellow en Medicina Materno-Fetal, Fundación Universitaria de Ciencias de la Salud, Hospital de San José, Bogotá, D.C., Colombia.

Institución: Unidad de Medicina Materno Fetal y Fetoscopia, Clínica Colsubsidio Orquídeas y Unidad de Diagnóstico y Terapia Fetal. Hospital de San José, División de Medicina Materno-Fetal, Departamento de Obstetricia y Ginecología, Fundación Universitaria de Ciencias de la Salud, Bogotá, D.C., Colombia. 


\begin{abstract}
Fetal death syndrome is one third of all fetal and infant mortality by over $50 \%$ of all perinatal deaths in developed countries. The causes of fetal death are rarely reported and most of them are unknown. This article intends to review and to understand the pathophysiology of the entity, to assess risk factors and to try to give our patients the possibility of a successful pregnancy.
\end{abstract}

A review the literature was made according to the PubMed database, EBSCO, Proquest and Ovid from 2000 to 2008.

Despite the significant decrease in fetal deaths in the last decades, fetal death syndrome remains a serious public health problem not only because it is a prevalent condition, but by underreporting and the lack of reliable reporting

\section{Key words}

Fetal death, fetal demise, stillbirth.

\section{Introducción}

El síndrome de muerte fetal se define como la muerte del feto que se presenta después de la semana 20 de gestación, en ausencia de trabajo de parto instaurado[1]. Constituye, en general, un tercio de toda la mortalidad fetal e infantil y más del $50 \%$ de todas las muertes perinatales en los países desarrollados. Las causas de muerte fetal raramente se reportan y la mayoría se desconoce. De acuerdo con lo anterior, es importante la identificación del riesgo antenatal con el fin de detectar por primera vez el antecedente, la enfermedad o la alteración que aumenta la posibilidad de muerte fetal, para determinar el riesgo relativo de resultados obstétricos adversos, al compararla con la población general[1,2].

La evaluación del riesgo por sí sola, parece ser cada vez menos útil en la predicción de complicaciones en la gestación pues, aun sin importar los riesgos calculados, 90 a $95 \%$ de los embarazos dan como resultado un recién nacido vivo y sano, lo cual contrasta con un gran número de óbitos fetales que ocurren en embarazos identificados como de bajo riesgo[3]. A pesar de haber disminuido dramáticamente sus tasas de presentación en los últimos 40 años, todavía se presenta gran dificultad en la evaluación y en la identificación de factores de riesgo[3, 4], como obesidad, ser el primer parto, edad materna avanzada en la primera gestación y las gestaciones múltiples.

Infortunadamente, las estadísticas mundiales no son comparables, pues en algunos países se incluyen pérdidas muy tempranas de la gestación y en otros hay pobre o deficiente registro e información, como ocurre en la mayoría de países subdesarrollados. Aunque los sistemas de registro no siguen criterios de inclusión uniformes, se estima que por cada 1.000 nacimientos se presentan 5,3 (rango, 4,2 a $6,8)$ muertes fetales en los países desarrollados y 25,5 (rango, 20 a 32 ) en los países en desarrollo[5, 6]. 
Se pretende con este artículo hacer una revisión que permita entender la fisiopatología de la entidad, evaluar los factores de riesgo y tratar de brindar a nuestras pacientes la posibilidad de un embarazo exitoso.

\section{Definición}

Un óbito fetal se define como la muerte en el útero del feto en desarro1lo, después de la semana 20 ó de fetos con peso mayor de $500 \mathrm{~g}$, lo cual correspondería a un feto de 22 semanas de gestación, lo anterior, en caso de no conocerse la edad de gestación por otro método[7, 8]. El feto no muestra signos de vida al nacimiento, ni responde a las maniobras de reanimación y no corresponde a una terminación inducida del embarazo[9]. Es de anotar que, de acuerdo con los reportes del Departamento Administrativo Nacional de Estadística de Colombia (DANE), la edad de gestación que se debe considerar es de 22 semanas, lo cual concuerda internacionalmente con el peso descrito.

La definición del DANE para muertes in útero es como sigue:

"muerte de un producto de la concepción, antes de su expulsión o extracción completa del cuerpo de su madre, independientemente de la duración del embarazo; la muerte está indicada por el hecho de que después de la separación, el feto no respira ni da ninguna otra señal de vida, como latidos del corazón, pulsaciones del cordón umbilical o movimientos efectivos de los músculos de contracción voluntaria" (http://www.dane.gov.co/).

La muerte fetal es el resultado de la interacción de diferentes y complejos procesos fisiopatológicos que ocurren en la madre, el feto y la placenta[10], cuya contribución es variable[11]. Sin embargo, la causa debe considerarse como aquélla inicial capaz de causar la secuencia de eventos que desencadenan la muerte del feto[9] (figura 1).

Con frecuencia, la causa del óbito fetal es difícil de identificar y, aunque los porcentajes son variados según la serie en estudio, muchos quedan catalogados como inexplicados, aunque se haga una investigación exhaustiva de las causas potenciales[7], ya sea por no encontrar una relación causal o porque más de un factor contribuye significativamente, superponiéndose.

En cada caso individual los hallazgos pueden considerarse como factores de riesgo y causas al mismo tiempo, como sucede con la hipertensión y el retardo del crecimiento intrauterino, e incluso, muchas de estas condiciones pueden estar relacionadas con la pérdida aun sin desencadenarla[7]. El origen multifactorial de esta entidad no es discutible. 


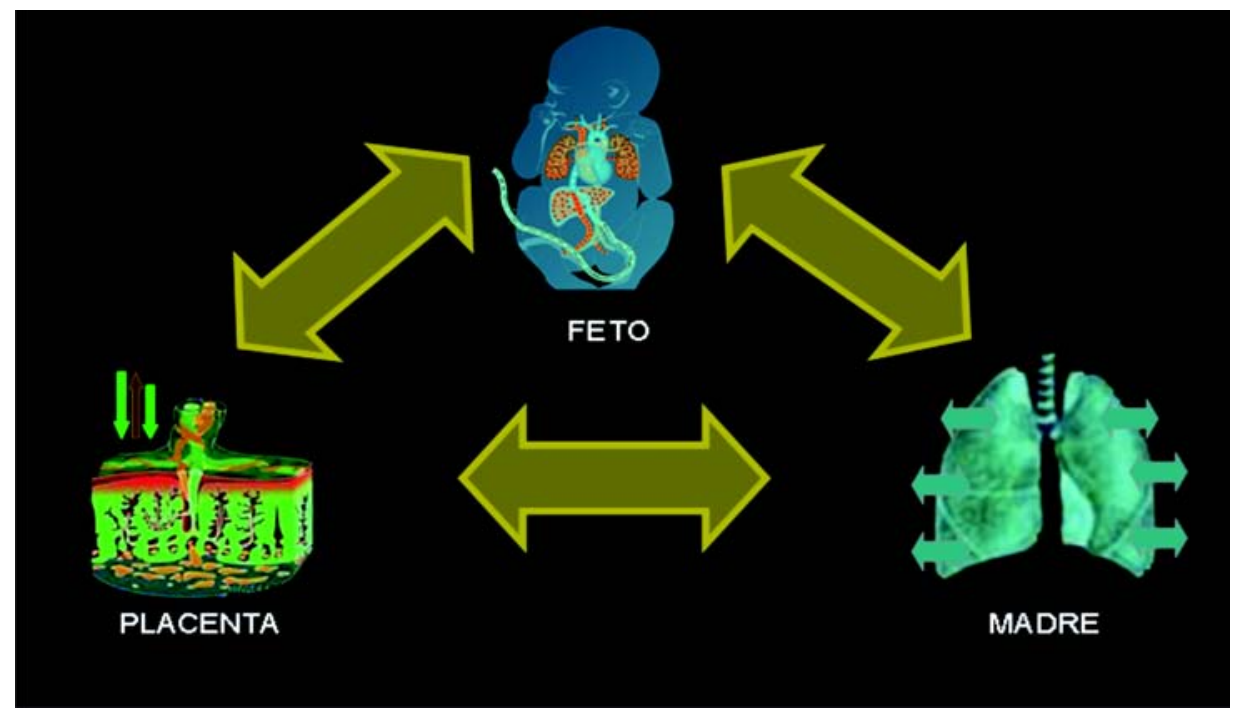

Figura 1. Relación madre-placenta-feto.

\section{Fisiopatología y clasificación}

La mayoría de las clasificaciones se han diseñado para usarse en países desarrollados con bajas tasas de óbitos fetales, uso liberal de la autopsia y registros de información de calidad razonable durante el embarazo, el parto y el periodo neonatal. En los países en desarrollo, la autopsia no se realiza de rutina; frecuentemente, los padres no autorizan el procedimiento; por lo tanto, en ocasiones, es difícil establecer la causa de muerte. Lo anterior se traduce frecuentemente en la carencia de datos médicos y la imposibilidad de realizar adecuadamente las siguientes asesorías a la pareja[12].

La clasificación de las muertes fetales en el útero continúa siendo un dilema, pues no se ha aceptado un único sistema de clasificación entre los muchos desarrollados[10]. Cada sistema posee diferentes fortalezas y debilidades al analizarlas de manera individual[7]. Sin embargo, es complicado tratar de comparar los diferentes sistemas de clasificación o hacerlos equiparables, debido a la gran variación en las definiciones de óbito fetal utilizadas y porque muchos sistemas de clasificación se diseñaron para "muerte perinatal", incluyendo casos de muerte neonatal temprana.

El primer intento de clasificación de las muertes fetales en el útero fue el de Aberdeen, que seguía criterios clínico-patológicos. Sin embargo, la más utilizada actualmente es la publicada en 1980 por Wigglesworth — con 
algunas modificaciones- que incluye nueve categorías[13] (tabla 1). Se han desarrollado sistemas más modernos, sin que hayan demostrado una verdadera utilidad científica.

Las muertes perinatales se clasifican según la causa presumible de muerte[14]. Sin embargo, la clasificación debe estar enfocada en la causa o condición obstétrica asociada. Hay más de 30 sistemas de clasificación reportados en la literatura; las más antiguas incluyen pocos subtipos, mientras que las más modernas buscan más información[6].
Aun así, no se ha logrado consenso sobre la importancia relativa de las diferentes causas[6]; se ha priorizado cada dato con criterios internos y externos, según tenga peso o actúe en cada caso en particular como causa o como factor de riesgo[12] pues, a excepción de algunas, son pocas las condiciones que cumplen criterios para ser capaces de causar inevitable e individualmente la muerte de un feto en el vientre materno, como lo hacen la muerte materna y el desprendimiento o abrupción total de la placenta[7].

De manera general y sencilla, se pueden clasificar según la edad de

Tabla 1. Clasificación de Wigglesworth (versión modificada) para muertes perinatales por causas obstétricas

\begin{tabular}{|c|c|c|c|c|}
\hline Anomalía congénita & $\begin{array}{l}\mathbf{1} \\
\mathbf{3} \\
\mathbf{5} \\
\mathbf{7}\end{array}$ & $\begin{array}{l}\text { Sistema nervioso central } \\
\text { Renal } \\
\text { Cromosómico } \\
\text { Otros }\end{array}$ & $\begin{array}{l}2 \\
4 \\
6\end{array}$ & $\begin{array}{l}\text { Sistema cardiovascular } \\
\text { Alimentario } \\
\text { Bioquímico }\end{array}$ \\
\hline Isoinmunización & 8 & Isoinmunización Rhessus & 9 & Soinmunización No-Rhessus \\
\hline Toxemia & 10 & Severa & 11 & Otra \\
\hline Hemorragia anteparto & $\begin{array}{l}12 \\
14\end{array}$ & $\begin{array}{l}\text { Abrupcio } \\
\text { Otra }\end{array}$ & 13 & Placenta previa \\
\hline Mecánico & $\begin{array}{l}15 \\
17\end{array}$ & $\begin{array}{l}\text { Pelvis } \\
\text { Otra mecánica }\end{array}$ & 16 & Prolapso de cordón \\
\hline Desorden materno & $\begin{array}{l}18 \\
20 \\
22\end{array}$ & $\begin{array}{l}\text { Trauma materno } \\
\text { Diabetes } \\
\text { Otras }\end{array}$ & $\begin{array}{l}19 \\
21\end{array}$ & $\begin{array}{l}\text { Hipertensión arterial } \\
\text { Cirugías abdominales }\end{array}$ \\
\hline Misceláneo & 23 & Especificar & & \\
\hline Inexplicado & $\begin{array}{l}24 \\
26\end{array}$ & $\begin{array}{l}<2500 \mathrm{~g}-<37 \text { semanas } 25 \\
<2500 \mathrm{~g}->37 \text { semanas } 27\end{array}$ & & $\begin{array}{l}500 \mathrm{~g}-<37 \text { semanas } \\
500 \mathrm{~g}->37 \text { semanas }\end{array}$ \\
\hline
\end{tabular}


gestación, en muertes fetales tempranas o difíciles de evitar (20 a 28 semanas de gestación) que corresponden a $50 \%$ de los óbitos, y tardías o potencialmente prevenibles (mayor de 28 semanas) [6, 7], teniendo en cuenta cuáles fetos pudieran ser viables según la edad de gestación y cuáles no[8].

Aunque la mayoría de óbitos ocurre antes de término y sólo $20 \%$ cerca al término, el riesgo de muerte aumenta con la edad de gestación, según Yudkin et al., quienes proponen el cálculo teniendo en cuenta el número de fetos intrauterinos vivos para cada edad de gestación y no el total de embarazos[15].

La causa de muerte difiere según la edad de gestación. En general, a medida que la pérdida es más temprana, se relaciona más con condiciones de origen materno y, si se da más tarde, está más relacionada con factores que afectan directamente al feto[9].

Así como la causa de la muerte fetal varía con la edad de gestación, la proporción de muertes fetales sin causa conocida a pesar de la exhaustiva investigación aumenta con la edad de gestación. Hasta la semana 27, la mayoría de las muertes, en especial en fetos con pesos menores de $1.000 \mathrm{~g}$, están relacionadas con infección, lo cual no ha disminuido de manera significativa ni siquiera con el uso de antibióticos; le siguen causas como el desprendimiento prematuro de placenta y las malformaciones letales. No obstante, alrededor de $21 \%$ son inexplicadas, mientras que, después de la semana 28 , se consideran inexplicadas hasta en $60 \%$ [8].

Algunas causas específicas de muerte fetal han cambiado notablemente en las últimas décadas, en especial en la segunda mitad del siglo $\mathrm{XX}$, con el desarrollo de estrategias para intervenirlas, lo cual es más evidente en los países desarrollados (tabla 2). Desde la aparición y utilización profiláctica de la inmunoglobulina $\mathrm{D}$ y de la monitorización fetal, se disminuyeron en $95 \%$ las muertes por causa de la isoinmunización y la asfixia durante el parto, respectivamente[6, 8]. De manera similar aunque en menor escala, se ha disminuido la mortalidad fetal en pacientes con diabetes e hipertensión, por el manejo cada vez más agresivo de estas condiciones, y se han modificado las tasas de óbitos con anomalías congénitas por la tamización temprana de alteraciones cromosómicas y no cromosómicas[8], sumada a la práctica de la interrupción voluntaria del embarazo.

Más de la mitad de los casos de óbitos fetales son inexplicados, sin importar el sistema de clasificación que se utilice y a pesar de que se realice un análisis anatomopatológico post mórtem[16, 17]. Este diagnósti- 
Tabla 2. Diferencias en condiciones asociadas a óbito fetal según el nivel de desarrollo

\section{Países desarrollados}

- Anomalías congénitas

- Restricción de crecimiento

- Enfermedades médicas maternas

- Desórdenes hipertensivos - preeclampsia

- Infecciones congénitas

$\sim$ Parvovirus B19

$\sim$ Str. B hemolítico

- Gestantes fumadoras

- Gemelares

co causal es muy poco útil al intentar brindar una asesoría a la madre para embarazos futuros o al intentar reforzar la atención en salud en puntos específicos de gran necesidad, así como para determinar las prioridades en el cuidado perinatal y la investigación para disminuir las tasas de mortalidad perinatal[17]. Los esfuerzos deben encaminarse a buscar la información suficiente en cada caso y contar con una clasificación que minimice las tasas de muerte de causa inexplicada y que considere esa aseveración como un diagnóstico de exclusión[8].

La mayoría de las causas de muerte fetal diferentes de anomalía congénita y la infección, está relacionada con la alteración de la placentación y con la función placentaria. En muchos casos, la causa directa del óbito fetal se relaciona con alteraciones placentarias en la interfase de las circulaciones materna y fetal e hipoper-

\section{Países en desarrollo}

- Distosias del parto

- Infecciones congénitas

$\sim$ Sífilis

$\sim$ Gram negativos

- Desórdenes hipertensivos - preeclampsia

- Desnutrición

- Antecedente de óbito fetal

- Anomalías congénitas

- Malaria

- Anemia falciforme

fusión, con posterior alteración en la autorregulación fetal [18].

Muchas de las muertes fetales no explicadas están relacionadas con retardo del crecimiento intrauterino, tanto a término como antes de término[16, 19]; además, se ha encontrado que los fetos pequeños para la edad de gestación tienen 10 veces más riesgo de muerte en el útero que los fetos con peso adecuado[8]. Tan sólo $25 \%$ de los retardos de crecimiento asociadas a muerte se detectan antenatalmente e, incluso, la detección puede ser cercana al $15 \%$ si la gestación ha sido considerada de bajo riesgo[16, 19]. Por lo tanto, la vigilancia del crecimiento fetal y el diagnóstico temprano de las alteraciones de las curvas de crecimiento son esenciales y deben hacer parte de las estrategias efectivas para prevenir las muertes fetales, haciendo que se puedan evitar o advertir. 
Los hallazgos del crecimiento fetal se relacionan con los del Doppler de las arterias uterinas y de las arterias umbilicales, en el que encontrar resistencias elevadas al flujo sanguíneo se relaciona con complicaciones obstétricas, entre las que se encuentran el retardo del crecimiento intrauterino y la muerte fetal[6].

La madurez placentaria ha sido usada como factor predictor de pérdida fetal, pero no hay estudios suficientes que confirmen o desmientan su utilidad, mientras que los resultados negativos presentan dificultades de interpretación y, por lo tanto, pierden valor en embarazos de poblaciones no seleccionadas[6].

Igualmente, se ha intentado usar los marcadores serológicos de aneuploidía del primero y el segundo trimestres como factores de predicción con base en la placentación inadecuada. Como factores de predicción se han calificado mejor la proteína placentaria asociada al embarazo tipo A y la alfafetoproteína, las cuales no se utilizan de rutina en población no seleccionada para calcular el riesgo y cuya utilidad clínica está siendo evaluada en la práctica[6].

Aun conociendo la fisiopatología de las pérdidas tardías de gestación, no hay un tratamiento de rutina que parezca ser efectivo para su prevención. Se ha intentado dar suplemento de ácido fólico en grandes dosis, en pacientes con la mutación C677T; en pacientes con trombofilias, se administran heparinas de bajo peso molecular cuando hay riesgo de enfermedad tromboembólica e, incluso, es administrado oxígeno suplementario materno con base en la hipoxia fetal como factor desencadenante; esto último necesita estudios de mayor poder estadístico para confirmar o negar su utilidad y ser recomendado para uso clínico[6].

\section{Factores de riesgo}

Son muchos los factores asociados a los desenlaces adversos de la gestación, en especial, los factores socioambientales y la enfermedad médica materna[20], especialmente la hipertensión arterial y la diabetes mellitus dependiente de la insulina, en las cuales se ha encontrado una relación con hasta la mitad de los óbitos fetales[21].

A pesar de la dificultad para obtener información certera por el subregistro y la gran cantidad de casos manejados como inexplicados, se han hecho esfuerzos por conocer las tasas de presentación y los riesgos relativos de la población afectada, con el propósito de mejorar la consejería y ofrecer la mejor atención a estas pacientes (tabla 3).

Factores de riesgo como el número de embarazos, la raza, la edad, la obesidad, el estrato socioeconómico, el ni- 
Tabla 3. Tasa de óbitos fetales según la condición médica asociada

\begin{tabular}{|c|c|c|c|}
\hline Condición materna & Tasa* & OR & Autor(es) \\
\hline $\begin{array}{l}\text { Embarazo } \\
\text { Embarazos de bajo riesgo } \\
\text { Embarazos múltiples } \\
\text { Antecedente de RCIU } \\
\text { Antecedente de óbito }\end{array}$ & $\begin{array}{r}6 \text { a } 7 \\
4.0-5.5 \\
12.0-30.0 \\
12.0-30 \\
9.0-20\end{array}$ & $\begin{array}{r}1.0 \\
0.86 \\
1.0-2.8 \\
2-4.6 \\
1.4-3.2\end{array}$ & $\begin{array}{l}\text { Alessandri } \\
\text { Fretts } \\
\text { Fretts } \\
\text { Fretts } \\
\text { Fretts }\end{array}$ \\
\hline $\begin{array}{l}\text { Desórdenes hipertensivos } \\
\text { - Hipertensión arterial (HTA) crónica } \\
\text { - HTA crónica + Preeclampsia sobreagregada } \\
\text { - Hipertensión inducida por embarazo } \\
\text { - Preeclampsia leve } \\
\text { - Preeclampsia severa } \\
\text { - HELLP } \\
\text { - Eclampsia }\end{array}$ & $\begin{array}{r}25 \\
52 \\
9 \\
5 \\
21 \\
50 \\
18\end{array}$ & $\begin{array}{l}1.2-4.0 \\
1.8-4.4\end{array}$ & $\begin{array}{l}\text { Ananth - Fretts } \\
\text { Mabie } \\
\text { Ananth } \\
\text { Sibai } \\
\text { Martin } \\
\text { Martin } \\
\text { Ananth }\end{array}$ \\
\hline $\begin{array}{l}\text { Diabetes } \\
\text { - Pregestacional } \\
\text { Diabetes tipo } 1 \\
\text { Diabetes tipo } 2 \\
\text { - Gestacional }\end{array}$ & $\begin{array}{r}10 \\
6 \\
35 \\
5\end{array}$ & $\begin{array}{r}1.7-7.0 \\
1.2-2.2\end{array}$ & $\begin{array}{l}\text { Landon } \\
\text { Cundy } \\
\text { Cundy } \\
\text { Landon }\end{array}$ \\
\hline $\begin{array}{l}\text { Obesidad } \\
\text { Lupus eritematoso sistémico (LES) } \\
\text { - LES activo }\end{array}$ & $\begin{array}{r}15-20 \\
40-150 \\
67\end{array}$ & $\begin{array}{r}2.1-2.8 \\
6.0-20\end{array}$ & $\begin{array}{l}\text { Stephansson } \\
\text { Le Thi Huong } \\
\text { Estes - Mintz }\end{array}$ \\
\hline $\begin{array}{l}\text { Trombofilias } \\
\text { Enfermedad renal crónica } \\
\text { - Compromiso renal leve } \\
\text { - Compromiso renal moderado a severo } \\
\text { Cunnigham }\end{array}$ & $\begin{array}{r}18-40 \\
15 \\
32-200\end{array}$ & $\begin{array}{r}2.8-5.0 \\
2.2-30\end{array}$ & $\begin{array}{l}\text { Davidson } \\
\text { Jungers - }\end{array}$ \\
\hline $\begin{array}{l}\text { Alteraciones tiroideas } \\
\text { Hipertiroidismo controlado } \\
\text { Hipertiroidismo no controlado } \\
\text { Hipotiroidismo } \\
\text { Hipotiroidismo subclínico }\end{array}$ & $\begin{array}{r}0-36 \\
100-156 \\
15-125 \\
0-15\end{array}$ & $2.2-3.0$ & $\begin{array}{l}\text { Alsulyman } \\
\text { Gaudet } \\
\text { Fisk } \\
\text { Davis }\end{array}$ \\
\hline
\end{tabular}

TASA $^{*}=$ Óbitos fetales por 1000 nacidos vivos de madres con la misma condición .

vel educativo, las condiciones médicas, el acceso a servicios de salud de calidad y las complicaciones obstétricas, hacen variar el riesgo de una mujer de tener un óbito fetal[6, 22]. Algunos de estos factores son modificables y sobre ellos se debe trabajar desde las etapas previas a la concepción. 
La historia de un óbito fetal previo se ha relacionado con efectos obstétricos adversos, que incluyen el daño neurológico y nuevas muertes perinatales[21]. Igualmente, las mujeres con embarazos complicados que resultaron en nacimientos de niños vivos y aquéllas con un primer embarazo complicado finalizado por cesárea, debido a un alto riesgo por enfermedad de origen vascular ( $\sin$ que sea la cesárea un marcador de otras condiciones médicas asociadas), tienen mayor riesgo de tener muertes fetales en los embarazos siguientes[6].

Las anomalías de la anatomía o de la longitud del cordón umbilical y su torsión, se han relacionado con el óbito fetal tras análisis retrospectivos de probabilidades; sin embargo, se desconocen las tasas reales de muerte fetal in utero en función de estas alteraciones[23, 24].

\section{Raza}

Las mujeres de los Estados Unidos de raza negra (afroamericanas) han mostrado tener dos veces más riesgo para óbito fetal. Aun sin ajustar estas cifras con las diferencias obstétricas y socioeconómicas, también tienen mayor tendencia a presentar diabetes, hipertensión y obesidad, y con ellas, ruptura prematura de membranas, desprendimiento placentario $\mathrm{y}$, por ende, muerte fetal[8].

\section{Estrato socioeconómico bajo}

El nacer en un país en desarrollo se considera un factor de riesgo para muerte fetal; además, se considera que la mayoría de las causas son prevenibles, como sífilis o paludismo, y se relacionan con obstáculos en la prevención, el acceso a servicios de calidad y la atención mediadas por factores sociales, económicos, geográficos, culturales, políticos y gubernamentales $[6,8]$.

Aun sin tener en cuenta el desarrollo del país, las pacientes de estratos más bajos tienen menor acceso a la educación, a una adecuada alimentación con altos índices de desnutrición y no buscan tempranamente atención prenatal, lo cual aumenta sus riesgos relativos de pérdidas fetales[8].

\section{Edad materna avanzada}

Se considera un factor de riesgo independiente para óbito fetal, aun considerando que estas mujeres, por su edad, tienen una mayor tendencia a padecer condiciones médicas asociadas que, a su vez, están relacionadas con mayores tasas de efectos perinatales adversos [8, 25].

La edad materna es un factor de riesgo importante para malformaciones congénitas, especialmente, si se asocian con factores genéticos. Con el 
diagnóstico temprano de las anomalías congénitas, las muertes fetales asociadas se han reducido. Sin embargo, esta mortalidad se ha trasladado hacia las primeras 20 semanas de gestación, por cuenta de las interrupciones voluntarias[6]. Algunos autores han descrito el factor vascular e inmunológico ligado a la edad como un factor determinante en la génesis de las alteraciones placentarias que podrían llevar a la muerte fetal[6, 7].

\section{Obesidad}

La prevalencia de la obesidad en la población está actualmente en aumento, compromete entre el 20 y $40 \%$ de las mujeres en edad fértil y se ha convertido en un serio problema de salud pública[20].

En las mujeres obesas, el riesgo de óbito fetal se ha relacionado con factores de comportamiento, socioeconómicos y obstétricos, pero también, con el aumento del índice de masa corporal (IMC) de manera independiente $[8,20]$.

Al parecer, la mujeres con alto IMC tienden a percibir menos los movimientos fetales y, por lo tanto, no mantienen un seguimiento constante de sus fetos; muchas son hiperlipidémicas, lo cual causa alteraciones endoteliales y, con ellas, agregación plaquetaria y aterosclerosis[8].

En estudios pequeños también se ha reportado que, durante el sueño, las mujeres gestantes obesas presentan más episodios de ronquidos, apnea del sueño y disminución de la saturación de oxígeno, lo cual se ha relacionado con mayor hipertensión inducida por el embarazo y restricción del crecimiento intrauterino[8].

\section{Trombofilias}

La relación de las trombofilias está mejor definida en las pérdidas fetales tardías que en las tempranas, aunque aún el conocimiento es muy limitado.

La predisposición genética está sustentada por la recurrencia del fenómeno, por ejemplo, en pacientes con enfermedades con mutaciones protrombóticas.

No se dispone de estudios amplios ni que analicen de manera separada cada defecto o mutación, pero se han relacionado con hallazgos placentarios anómalos en alrededor de 75 a $80 \%$ de los casos en la evaluación post mórtem[8, 20].

\section{Lupus eritematoso sistémico}

A pesar de afectar menos del $1 \%$ de las mujeres en edad fértil, el aumento del riesgo de óbito fetal es desproporcionadamente alto, en especial, en aquéllas con enfermedad renal preexistente, hipertensión arterial o presencia de anticoagulante lúpico positivo. 
El paso transplacentario de anti-Ro y anti-La compromete el 5\% de los fetos y se relaciona con un mal resultado obstétrico, por el riesgo de bloqueo cardiaco fetal que puede resultar en hydrops no inmune de origen cardiaco y muerte.

Los riesgos se mantienen, aun cuando la concepción ocurra durante periodos de inactividad de la enferme$\operatorname{dad}[8,20]$.

\section{Hipertensión arterial}

Las muertes relacionadas con la hipertensión arterial sistémica cada vez son más infrecuentes, gracias a la mejor atención médica y obstétrica; se relacionan con los estados más graves y obedecen a complicaciones como desprendimiento prematuro de placenta, hemorragia materna, insuficiencia o infarto placentario[8, 20].

\section{Diabetes mellitus}

Con la introducción de la insulina en la práctica clínica, la disminución de las muertes fetales relacionadas con esta enfermedad ha sido significativa. Actualmente, la muerte fetal relacionada con la diabetes mellitus ocurre con más frecuencia en el tercer trimestre del embarazo en pacientes con poco control de la glucemia y obedece a complicaciones como macrosomía, polihidramnios, retardo del crecimiento intrauterino y preeclampsia.
Las complicaciones son más frecuentes si la diabetes se presenta antes de la gestación que cuando lo hace durante ella; en este último caso, puede llegar a tener riesgos relativos similares a los de la población sana, si se mantiene un control adecuado de la glucemia, si se hace control prenatal y si hay una adecuada definición del tiempo de parto[8, 20].

\section{Falla renal}

En estas pacientes, el pronóstico del embarazo está dado principalmente por el grado de compromiso renal durante la concepción; es más sombrío en cuanto más grave sea la falla orgánica.

En caso de acompañarse con anemia, hipertensión o el desarrollo de preeclampsia, disminuye la posibilidad de tener resultados obstétricos favorables, al igual que en aquellas pacientes que requieren diálisis.

Sin embargo, las opciones de tener resultados favorables aumentan tras el trasplante renal y la normalización de las condiciones basales[20].

\section{Infección}

La infección se encuentra muy relacionada con las tasas de muerte perinatal $\mathrm{y}$, aunque se han modificado las estrategias para la prevención y el diagnóstico temprano[8], no han cambiado mucho las expectativas de 
vida de estos fetos porque en su mayoría se afectan antes de término.

Se ha demostrado que los procesos inflamatorios se relacionan con resultados obstétricos adversos, predominantemente, en el feto pretérmino. Actualmente, se sabe que la respuesta inflamatoria fetal asociada a infección clínica o subclínica es capaz de alterar el intercambio gaseoso, causar hipoxia fetal y, así, disminuir la supervivencia[26, 27].

\section{Embarazos múltiples}

Las tasas de óbitos fetales en los embarazos múltiples se encuentran cuadriplicadas en comparación con las de embarazos únicos y se relacionan con complicaciones específicas de este tipo de embarazos, que se hacen mayores en los embarazos con mayor número de fetos. Contribuyen de manera importante al total de las tasas de muertes fetales y van de la mano con el aumento de 6 a 12 veces que han experimentado los embarazos múltiples secundarios, a su vez, a la mayor edad de las madres y a las técnicas de fertilización in vitro[6,8].

\section{Seguimiento de las pacientes en riesgo}

El uso de las pruebas de bienestar fetal, más que modificar el riesgo de óbito fetal, muestra ciertas condiciones clínicas que indican tomar deci- siones en cuanto al tiempo y la vía del parto[6], lo cual, a su vez, puede influir en la disminución de las pérdidas fetales pero podría aumentar la morbimortalidad materna y neonatal asociadas a un aumento de las intervenciones obstétricas[8].

Se propone unificar los algoritmos de seguimiento de estas pacientes según la enfermedad de base, de manera que se evite la sobreestimación del riesgo y permita actuar de manera oportuna.

La presentación de un óbito fetal es indicación absoluta de valoración anatomopatológica de la placenta, pues esto puede dar luces sobre las causas. En $40 \%$ de los casos, la valoración anatomopatológica brinda nueva información y en $25 \%$ da información diferente a la capturada clínica y ecográficamente[28]. Para muchos padres es la única forma de averiguar qué ocurrió y delinear el plan de manejo de futuros embarazos, además que puede ayudar a la resolución de conflictos médico-legales.

\section{Discusión}

A pesar de la disminución significativa de los óbitos fetales en las últimas décadas, sigue siendo un serio problema de salud pública, no sólo por ser una condición prevalente, sino por el subregistro y la ausencia de reportes confiables que tengan en cuenta tanto 
el número de casos como la precisión en la determinación de su causa.

Es poco el conocimiento que se tiene sobre la fisiopatología de la muerte fetal en relación con cada uno de los factores desencadenantes y, por esta razón, se ha fallado en clasificarla en forma consistente, aun cuando existen múltiples clasificaciones en la literatura.

Esta desinformación hace que sea muy difícil estimar los riesgos de la población y, por lo tanto, delinear planes para el mejoramiento que lleven a la prevención y a la disminución de este resultado adverso de la gestación. La asesoría del embarazo actual, la valoración del riesgo individual y la preparación para embarazos futuros según los antecedentes, son pilares fundamentales de la consulta prenatal y del control prenatal establecido, con el fin de informar adecuadamente a las madres y a sus parejas sobre las posibilidades del desenlace de todos los embarazos muchas veces considerados como normales.

Se debe trabajar en pro de lograr la unificación de una clasificación adecuada relacionada con la fisiopatología del evento, pero que esté enfocada en el área prenatal y permita incluir a la mayoría de las pacientes de manera confiable en uno de los grupos, dejando la categoría de inexplicable como diagnóstico de exclusión.
Una vez conocida la realidad de nuestra población, se deben identificar los factores que aumentan el riesgo y delinear sobre ellos planes de seguimiento y evaluación temprana.

Como conclusión, el síndrome de muerte fetal continúa siendo una condición de interés en el control de las pacientes obstétricas de bajo y alto riesgo. El conocimiento de su existencia, etiología y fisiopatología, permitirá un reconocimiento precoz de los factores de riesgo modificables $\mathrm{y}$, de esta manera, identificar los fetos en riesgo que son susceptibles de intervención. El manejo de estas pacientes debe realizarse en unidades maternofetales con experiencia. Los trabajos futuros deben enfocarse en conocer su prevalencia actual en nuestro medio.

\section{Bibliografía}

1. Molina-Giraldo S, Alfonso DA. Vigilancia fetal anteparto. Texto de perinatología y neonatologia. Bogotá, Colombia. Organización Mundial de la Salud (en prensa).

2. Gribbin C, James D. Assessing fetal health. Best Pract Res Clin Obstet Gynaecol. 2004;18:411-24.

3. Filkins, Karen; Koos, Brian J. Ultrasound and fetal diagnosis Current Opinion in Obstetrics and Gynecology. 2005 April; 17(2):185-95.

4. Jones N, Bugg G, Gribbin C, RaineFernning N. Assessing fetal health. Obstetrics, Gynecology and Reproductive Medicine. 2008;18:145-9. 
5. Smith G. Estimating risks of perinatal death. Am J Obstet Gynecol. 2005 Jan;192(1):17-22. Review.

6. Smith G, Fretts R. Stillbirth. Lancet. 2007;370:1715-25.

7. Silver RM, Varner MW, Reddy U, Goldenberg R, Pinar H, Conway D, Bukowski R, Carpenter M, Hogue C, Willinger M, Dudley D, Saade G, Stoll B. Work-up of stillbirth: A review of the evidence. Am J Obstet Gynecol. 2007 May;196(5):433-44.

8. Fretts R. Etiology and prevention of stillbirth. Am J Obstet Gynecol. 2005;193:1923-35.

9. Martin J, Hoyert D. The National Fetal Death File. Semin Perinatol. 2002; 26:3-11.

10. Vergani P, Cozzolino S, Pozzi E, Cuttin MS, Greco M, Ornaghi S, Lucchini V. Identifying the causes of stillbirth; a comparison of 4 classification systems. Am J Obstet Gynecol. 2008;199:319. E1-319.

11. Kean L. Intrauterine fetal death. Curr Obstet Gynaecol. 2006;16:199-205.

12. Elamin S, Langhoff-Roos J, Boedker B, Ibrahim SA, Ashmeig AL, Lindmark G. Classification of perinatal death in a developing country. Int J Gynaecol Obstet. 2003;80:327-33.

13. Galan-Roosen A, Kuijpers J, van der Satraaten P, Merkus J. Eur J Obstet Gynecol. 2002;103:30-6.

14. Gardosi J, Francis A, Cnattingius S. Stillbirth and fetal growth restriction at preterm and term gestations in singleton pregnancies: a multivariate analysis. Am J Obstet Gynecol. 2003;189: S158.

15. Cnattingius S, Stephansson O. The epidemiology of stillbirth. Semin Perinatol. 2002;26:25-30.

16. Gardosi J, Badger S, Tonks A, Francis A. "Unexplained" stillbirths: An investigation of the clinically relevant con- ditions at the time of fetal death. Am $J$ Obstet Gynecol. 2003;189:S158.

17. Kady S, Gardosi J. Perinatal mortality and fetal growth restriction. Best Pract Res Clin Obstet Gynaecol. 2004;18: 397-410.

18. Grafe M, Kinney H. Neuropathology associated with stillbirth. Semin Perinatol. 2002;26:83-8.

19. Ongu S, Danielian P. Unexplained stillbirths: Are they preventable? Int J Gynaecol Obstet. 2000;69:159-61.

20. Simpson L. Maternal medical disease: risk of antepartum fetal death. Semin Perinatol. 2002;26:42-50.

21. Weeks J, Asrat T, Morgan M, Nageotte BM, Thomas SJ, Freeman RK. Antepartum surveillance for a history of stillbirth: when to begin? Am J Obstet Gynecol. 1995;172:486-92.

22. Reddy U, Ko CW, Willinger M. maternal age and the risk of stillbirth throughout pregnancy in the United States. Am J Obstet Gynecol. 2006;195: 764-70.

23. Molina-Giraldo S. Circulares de cordón: un dilema obstétrico. Controversias en ginecología y obstetricia. 2006;16:2680-95.

24. Collins J. Umbilical cord accidents: human studies. Seminars in Perinatology. 2002;26:79-82.

25. Bateman B, Simpson L. Higher rate of stillbirth at the extremes of reproductive age: a large nationwide sample of deliveries in The United States. Am J Obstet Gynecol. 2006;194:840-5.

26. Fifer W, Myers M. Sudden fetal and infant deaths: shared characteristics and distinctive features. Semin Perinatol. 2002;26:89-96.

27. Gibbs R. The origins of stillbirth: infectious diseases. Semin Perinatol. 2002;26:75-8.

28. Yee Khong T. The placenta in stillbirth. Current Diagnostic Pathology. 2006;12:161-72. 